\title{
Animal Models as Tools for Translational Research: Focus on Atherosclerosis, Metabolic Syndrome and Type-II Diabetes Mellitus
}

\author{
Isaac Karimi
}

Additional information is available at the end of the chapter

http://dx.doi.org/10.5772/47769

\section{Introduction}

Close to one century ago, Joslin, an American diabetologist, proposed the link between diabetes and obesity [1]. He concluded that "diabetes is largely a penalty of obesity, and the greater the obesity, the more likely is Nature to enforce it". In the 1950s, Vague [2] described that central obesity predisposes not only to diabetes but also to atherosclerosis. In the 1970s, for the first time, Haller [3] used the term "metabolic syndrome" (MetS) for associations of obesity, diabetes mellitus (DM), hyperlipoproteinemia, hyperuricemia, and hepatic steatosis when describing the additive effects of risk factors on atherosclerosis. Phillips developed the concept of metabolic risk factors for myocardial infarction and described a cluster of abnormalities including glucose intolerance, hyperinsulinemia, hyperlipidemia, and hypertension [4,5]. In 1988, Reaven, an American endocrinologist, propounded that insulin resistance (IR) was the cause of glucose intolerance, hyperinsulinaemia, increased very-lowdensity lipoprotein cholesterol (VLDL-C), decreased high-density lipoprotein cholesterol (HDL-C) and hypertension and named the constellation of abnormalities "syndrome $X$ " [6]. Reaven did not include abdominal obesity, which has also been hypothesized as the underlying factor, as part of the condition. In the late 1990s and the early 21st century, MetS was widely recognized as a leading risk factor for cardiovascular morbidity and mortality and variously defined by World Health Organization [7], International Diabetes Federation (IDF [8]), the European Group for the Study of Insulin Resistance [9] and the National Cholesterol Education Program Adult Treatment Panel III [10] based on the reference intervals of its components. Accordingly to these definitions, MetS is thought to represent a combination of cardiometabolic risk determinants, including obesity, glucose intolerance and IR, dyslipidemia (including hypertriglyceridaemia, increased free fatty acids (FFAs) and decreased HDL-C) and hypertension and more recently a growing list of clinical 
manifestations like polycystic ovarian syndrome (PCOS), atherosclerosis, proinflammatory state, oxidative stress and non-alcoholic fatty liver disease (NAFLD) has been associated to it.

The MetS is increasingly recognized as a strong predictor of patient risk for developing coronary artery disease. It is associated with an atherogenic dyslipidemia characterized by elevated levels of triglycerides (TGs), reduced levels of HDL-C and a preponderance of small dense low-density lipoprotein (LDL) particles [11]. An atherogenic dyslipidemia is an integral component of MetS, and a major contributor to the cardiovascular risks in patients. These alarming situations increase the priority for developing new methods and technologies to investigate and to fight the MetS and its related comorbidities. Translational physiology offers us specific animal models for investigating these conditions to help support biomedical research efforts towards finding the necessary cures. This chapter summarizes various types of animal models that used as a tool in lipoprotein clinical researches and critically evaluates the physiological fidelity of these animal models to the human condition. The animal models are used to investigate biological or pathobiological phenomena or employed to find therapeutic and/or toxic effects of a xenobiotic or food ingredients. The laboratory animal models are developed and used to study the cause, nature, and cure of human lipoprotein disorders. They may conveniently be categorized in one of the following two groups:

1. Experimental animal models of lipoprotein disorders

2. Spontaneous animal models of lipoprotein disorders

\section{Experimental animal models of lipoprotein disorders}

Experimental (induced) models are healthy animals in which the condition (usually disease) to be investigated is experimentally induced, for instance, the induction of DM with encephalomyocarditis virus or alloxan. Although homologous animal models that completely show symptoms and the course of the lipoprotein metabolic disorders are very rare, the most induced models are exploratory, helping to understand mechanisms operative in fundamental normal biology or mechanisms associated with an abnormal biological function. Generally, induced models of metabolic disorders are prepared by genetic manipulation, dietary intervention, surgery, applying xenobiotics (drugs or toxins), and a combination of mentioned methods (see review [12]). This chapter will focus mainly on diet-induced and spontaneous animal models commonly used to investigate lipoprotein metabolic disorders. Readers referred to chapter 22 to study transgenic models of lipoprotein disorders.

Nowadays, obesity, particularly visceral (or central) obesity, is accepted as network backbone of the other MetS components and their manifestations. It has been reported that the incidence of MetS and type 2 diabetes (T2D) increases with the severity of obesity [13]. In this context, increasing body mass index is positively associated with prevalence of both impaired glucose tolerance and T2D and also correlated with dyslipidemia component of MetS that characterized by (a) increased flux of free fatty acids (FFA), (b) raised TGs values, 
(c) low HDL-C values, (d) increased small, dense low density lipoprotein particles (e) increased TC and LDL-C and (f) raised apolipoprotein (apo) B levels

The intake of high energy diet and sedentary behavior in developed countries has an accrual effect on the incidence of obesity. Although the association between visceral fat and MetS is strong, the mechanism is not fully elucidated. The adipose tissue is not an inert tissue and constitutively produces adipocytokines that involve in pathogenesis of MetS and IR (see review [14]). Diets play a fundamental role in inducing obesity-related diseases in human, and most animal models do use diet as a way to precipitate the obesity-related diseases. Today, most diet-driven animal disease models are generated using open source, purified ingredient diets. The "open source" nature of purified ingredient diets allows researchers to compare data from different studies, since the diet formulas are generally freely available to the public, while the "close source", chow diets are differently formulated. Purified ingredients, on the other hand, are highly refined and contain just a single nutrient (ie. fructose). These ingredients have little variability and therefore provide consistency between batches, and so help to minimize data variability. There are numerous differences between chows and purified diets, creating countless variables, thus making it difficult to interpret the results when these diets are used together in a study. Chow is a nonpurified diet composed of a mixture of intact feed. In contrast, purified diets provide macronutrients as purified ingredients. For example, carbohydrate in chow diets is derived from complex mixtures of corn and wheat flakes, wheat middlings, ground corn, and dried whey. In addition to carbohydrate, these ingredients provide variable amounts of protein, fat, vitamins, minerals, and various phytochemicals and other (anti)nutrients. Some of these compounds, in particular the phytoestrogens, may act as endocrine disruptors that alter endocrine milieu and disease progression and so are usually unwanted variables. Finally, purified ingredient diet formulas can be easily modified so that researchers can intentionally and specifically change one ingredient at a time, allowing them to study the effects of large or small changes in the nutritional quantity and quality of the diet. Because of these advantages, most metabolic disease animal research uses and requires purified ingredient diets. In addition to purified and chow diets, some scientists used what is known as the cafeteria diet (CAF) to induce obesity. In this model, animals are allowed free access to standard chow and water while concurrently offered highly pleasant, energy dense, unhealthy human junk foods including cookies, candy, cheese, and processed meats ad libitum. These foods contain a substantial amount of salt, sugar, and fat and are meant to simulate the human "Western diet". However, the nutritive and nonnutritive components of these foods are not well defined. In addition, the animal may choose a different selection of foods each day. In this section, I discuss how high diets influence the phenotypes of the obesity and/or MetS in translated animals.

\subsection{The mouse models}

The advantages of mouse models that made them suitable for translating human conditions include a well-known genome, relative ease of genetic manipulation, a short breeding span, access to physiological and invasive testing, short reproductive cycle, large litter size, much 
lower cost and possibility of conducting longitudinal studies using larger numbers of animals, rapid development of atherosclerotic plaques, only partial resemblance to humans, very high levels of blood lipids, useful for noninvasive imaging and large experience.

Normal mice have traditionally not been ideal models of cardiovascular disease research since they typically have very low levels of TC and LDL-C but high levels of HDL-C. This is in contrast to humans in whom the reverse is true because unlike humans and several other animals, mice do not possess plasma cholesteryl ester transfer protein (CETP) and, therefore, about $70 \%$ of the plasma TC is found in HDL particles. Mouse models have proved to be useful to study development and progression of atherosclerotic lesion, and several reviews have extensively discussed the different available models (see review [15]). The ability of mice to maintain their cholesterol profile even in the face of highcholesterol diets means that very little actual atherosclerosis develops [16]. As wild-type mice are resistant to lesion development, the current mouse models for atherosclerosis are based on genetic modifications of lipoprotein metabolism with additional dietary changes. In order to 'force' the atherosclerosis phenotype on normal mice, it is usually necessary to combine high concentrations of dietary cholesterol with $0.25 \%-0.5 \%$ cholic acid which promotes fat and cholesterol absorption from the intestine [17]. However, cholic acid can also promote liver inflammation, decrease bile acid production, and alter circulating TG and HDL-C, it may independently affect the development of atherosclerosis [15]. Atherosclerosis is a complex multifactorial disease with different etiologies that synergistically promote lesion development. High-fat diets (HFDs) are used to model obesity, dyslipidaemia, atherosclerosis, IR and MetS in rodents (see reviews $[18,19]$. High-fat diet (HFD) feeding in mice increased systolic blood pressure and induced endothelial dysfunction [20] and some kind of nephropathy [21]. Different types of HFDs have been used with fat fractions ranging between $20 \%$ and $60 \%$ energy as fat as either animal-derived fats, such as lard or beef tallow, or plant oils such as olive or coconut oil [22]. Long-term feeding of rats (60\% of energy) and mice (35\% fat wt/wt) with HFD increased body weight compared to standard chow-fed controls [23]. Although the increase in body weight was significant after as little as 2 weeks, the diet-induced phenotype became apparent after more than 4 weeks of HFD feeding [23]. Long-term feeding with both animal and plant fat-enriched diets eventually led to moderate hyperglycaemia and impaired glucose tolerance in most rat and mouse strains [24]. Lard, coconut oil and olive oil (42\% of energy content) increased body weight, deposition of liver TGs, plasma TGs and FFAs concentrations and plasma insulin concentrations [22]. Lard, coconut oil and olive oil caused hepatic steatosis with no signs of inflammation and fibrosis [22]. Although HFD induces most of the symptoms of human MetS in rodents, it does not resemble the diet causing MetS and associated complications, as the human diet is more complex than a HFD. Other major components of modern diets are refined carbohydrates and fructose. The epidemiologic data has proved that a significant correlation in the prevalence of diabetes with fat, carbohydrate, corn syrup (source of fructose), and total energy intakes. The striking features of these studies are the fact that intake of corn syrup was positively associated with T2D, while protein and fat were not (see review [25]). Most studies have utilized mice as animal models to define the role of 
carbohydrate enriched diets in formation of different aspects of MetS. The interested readers will have to go to the current literature in order to understand more fully the fidelity of mice for translation of similar conditions in humans. In this context, high-fat high-carbohydrate (HFHC) diet contains $55 \%$ fructose and $45 \%$ sucrose $(\mathrm{wt} / \mathrm{vol})$ in drinking water has been fed to nongenetically modified adult male C57Bl/6 mice for 16 weeks led to obesity and nonalcoholic steatohepatitis (NASH) [26]. HFHC has been used to induce hyperglycemia, glucose intolerance, IR, increased fat pad weight and adipocyte hypertrophy and commonly HFHC-fed mouse models used to screen therapeutic effects of various drugs and diets against MetS and its comorbidities (e.g., [27]). Charlton and colleagues recently proposed an obese mouse model of NASH that induced by feeding fast food (high SFs, cholesterol, and fructose) diet [28]. C57BL/6J mouse is T2D model by simply feeding HFD to nonobese, nondiabetic C57BL/6J mouse strain. It is characterized by marked obesity, hyperinsulinaemia, IR and glucose intolerance [29]. Diets contribute to T2D in mouse identical to human, and a HFD and sucrose administration appears to speed up the development of the disease in mice. In this context, impaired insulin secretion and/or impaired insulin action also contribute to the diabetic phenotype for these mice [30]. However, the mouse models are observed to develop diabetes in relation to profound obesity and do not display the same islet pathology as humans with T2D [31]. A large number of investigations also have been carried out within recent years, concerning the therapeutic action of various (bio)pharmaceutical and nutraceutical compounds on mouse models of lipoprotein disorders (e.g., [32]).

\subsection{The rat models}

Rats, like humans, showed different vulnerability to diet-induced obesity. At first, an animal model of diet-induced obesity is one introduced by Levin and coworkers [33] and developed into a purified diet model [34]. In this model, Sprague-Dawley (SD) rats fed a purified moderately high-fat (MHF) diet exhibit a bimodal pattern in body weight gain similar to that observed in humans. Approximately half of the rats gain weight rapidly compared with chow-fed rats (obesity prone [OP] or diet-induced obese [DIO]), whereas the other half gain BW at a rate similar to or lower than that of the chow-fed animals (obesity resistant [OR] or diet-resistant [DR]) [34-36]. Most rodents tend to become obese on HFD and very high-fat diet (VHFD), but there can be variable responses in glucose tolerance, IR, TGs, and other parameters depending on the strain and gender, and source of dietary fat $[22,37]$. When outbred SD and Wistar rats were placed on HFD (32 or $45 \mathrm{kcal} \%$ fat), there was a wide distribution in body weight gain and a subset of animals became obese, whereas others remained as lean as the animals fed with a low-fat diet (LFD) have shown that the rat model of diet-induced obesity develops mild hypertension accompanied by vascular and renal changes similar to those observed in obese hypertensive humans [35,38]. The MHF diet that they used contains $32 \% \mathrm{kcal}$ fat, a value similar to the average Western diet, as opposed to many other models that have very high levels of fat [38]. All rats fed the MHF diet did not become obese and their body weight displayed a bimodal distribution. The increased body weight reflects an increase in the adipose mass in the OP rats versus the chow-fed rats [38]. 
Elevation of plasma TGs and FFAs was commonly observed in patients with diabetic dyslipidemia or obesity [39]. Evidence showed that hyperglycemia and hypertriglyceridemia had direct effects on arterial wall and induced endothelial dysfunction [40]. The elevation of TGs and fasting plasma glucose was noted in HFD studies [41]. However, the levels of TGs and TC in high-fat fed DR rats were no more than chow-fed control rats. The HDL-cholesterol level decreases in hypercholesterolemic and MHF-fed rats [38]. The TGs content of plasma, LDL, and VLDL has been increased in OP rats fed MHF diet after 3 weeks [38]. As opposed to cholesterol content, this difference is even greater after 10 weeks of the MHF diet therefore authors concluded that factors other than diet like reduced growth hormone secretion are also responsible for the high levels of TGs in OP rats [38,42]. The underlying mechanism is not known. Insight into the differences in endocrine and lipoprotein metabolism may provide further evidences. For example, Yang et al's study showed that DR rats had higher levels of plasma peptide $Y Y$, a gut-derived anorexigen, than $\mathrm{DIO}$ and the control groups. This indicates that a difference in appetite control is responsible for the lower caloric intake and weight gain in DR rats [43]. One of the common features of obesity in humans is dyslipidemia which occurs in rat model of diet-induced obesity and is frequently associated with hypertension [38]. I have decided to ignore molecular mechanisms of hypertension in OP rat because of limited space. However, hypertension developed in OP, but not OR, rats, is a multifactorial disorder and diet is not the major factor that causes the high blood pressure in this model.

According to Barker hypothesis, adult metabolic diseases are programmed during fetal life [44]. To investigate the mechanisms by which altered intrauterine milieu predisposes to later development of MetS, different animal models have been developed (see review [45]). Interestingly, offspring of rats fed high saturated fats during pregnancy have fetal IR [46], abnormal cholesterol metabolism [47] and raised adult blood pressure [48]. Furthermore, the outbred Sprague-Dawley DIO and DR rats have been selectively bred over time such that their future body weight response to a HFD is known in utero, allowing the researcher to look early in life (prior to the onset of obesity) for genetic traits that may later predispose them to their DIO or DR phenotypes [37,49].

The inbred obese Zucker diabetic fatty (ZDF) rat is high-fidelity model with close resemblance to human case in obesity and T2D. The males become obese and diabetic on a LFD, but HFD feeding promotes more robust disease. The female ZDF rat is unique in that while they are obese, they do not develop diabetes unless fed a diet (in this case, chowbased) containing $48 \mathrm{kcal} \%$ fat [50]. The female ZDF rat is also suitable model mimics prediabetic state in humans because she shows a prolonged period of insulin sensitivity prior to the onset of diet-induced diabetes [51]. The ZDF rats show profound dyslipoproteinaemia with increased TC and TGs levels and lower chylomicra disposal rates that mimics conditions occurred in human case of obesity [52]. Although normal rats are not ideal model of cardiovascular disease research since they typically have very low levels of TC and LDL$\mathrm{C}$ but high levels of HDL-C, they are mild diet-responsive. The ability of rats to sustain their cholesterol profile even in the face of high-cholesterol diets means that very little actual atherosclerosis develops [16]. However, feeding Wistar rats a high calorie "Western diet" 
( $45 \%$ fat) for up to 48 weeks induces obesity and cardiac dysfunction, while a high fat diet (60\% fat) induces obesity only [53]. The "Western diet" composed of a purified ingredient SF-rich HFD, and cholesterol ( $\sim 0.2 \%$ by weight) can elevate TC and LDL-C and in turn cause atherosclerosis in certain rodent models and humans [54]. A mixture of high levels of dietary cholesterol with $0.25 \%-0.5 \%$ cholic acid has been used to induce atherosclerosis phenotype on normal rats and mice for many years ago [55]. More recently, Zaragosa and colleagues introduced various animal models of cardiovascular diseases (see review [56]). Surprisingly rat does not develop atheroma in the process of atherosclerosis (see review [56]). Generally rats are highly resistant to the development of atherosclerosis because they lack physiological resemblance on many aspects with humans that are pathophysiologically important [57]. For example, HDL is dominating lipoprotein in these animals and rat platelets are generally resistant in hyperlipidemic condition (see review [58]). Rats are potentially practical model for studying hypercholesterolemia along with hypertension (see review [58]). They exhibit augmented thrombotic response and develop coronary atherosclerotic lesions under hypertensive and hyperlipidemic conditions (e.g., [59]). Triglyceride-rich diets containing various amounts of cholesterol, with or without cholic acid have been used to induce hypercholesterolemia in rats. The fat sources vary from lard to soybean, canola or sunflower oils. Nevertheless, the question of the caloric value of the employed diets has not yet been considered properly since their high fat content, which is the strategy used in order to induce hypercholesterolemia, leads to lower ingestion by the animals and induces malnutrition. To overcome this shortcoming, Matos and colleagues [60] proposed a diet containing $25 \%$ soybean oil, $1.0 \%$ cholesterol, $13 \%$ fiber (cellulose) and $4,538.4 \mathrm{Kcal} / \mathrm{Kg}$ that led to an increase in LDL-C, a decrease in the HDL-C fraction and affected less the hepatic function of the rats during eight weeks. Roberts and colleagues presented a rat model of diet-induced syndrome $X$ and they explored potential mechanisms of hypercholesterolemia in diet-induced syndrome $X$ [61]. To induce syndrome $X$, female Fischer rats were fed a high-fat (primarily from lard plus a small amount of corn oil), refined-carbohydrate (sucrose) diet for 20 months [61]. Sampey and colleagues [62] have demonstrated that the CAF is a more robust model of MetS than lard-based HFD and that the rapid-onset of weight gain, obesity, multiorgan dysfunctions and pathologies observed in the CAF model more closely reflect the modern human condition of early onset obesity. However, they did not repot possible lipid-lipoprotein disorders that may be occurred in their model. Recently, Manting and colleagues [63] have shown that a combination of chronic stress and HFD (83.25\% basal feed, 10\% lard, 1.5\% cholesterol, $0.2 \%$ sodium taurodeoxycholate, $5 \%$ sugar and $0.05 \%$ propylthiouracil) can induce lipid metabolism disorder in Wistar rats and they claimed that their multiple factor model better mimics the disease characteristics of human beings.

\subsection{The hamster models}

Hamsters are another animal model can be used to assess some aspects of MetS. Like rats and mice, HDL-C is predominant plasma cholesterol-rich lipoprotein in these animal, but in contrast, dietary cholesterol $(\sim 0.1 \%)$ can significantly elevate LDL-C and like humans, SF 
can increase these levels further [64]. The combination of high dietary SF and cholesterol is commonly used to promote atherosclerosis in these animals and atherosclerotic lesions similar to those found in humans can be found after prolonged feeding periods [65]. Actually, cholesterol itself may not always be necessary for this phenotype, since a purified diet with no cholesterol but high concentrations of SF can promote more aortic cholesterol accumulation compared to a diet with both cocoa butter and $0.15 \%$ cholesterol [66]. Cholesterol-fed hamsters have been used to screen therapeutic anti-atherosclerotic and hypolipidemic properties of (phyto)medicines (e.g., $[67,68]$ ). Hamsters have been proposed as an animal model to evaluate diet-induced atherosclerosis since the 1980s [69]. Relative to other normal rodent models, hamsters have a low rate of endogenous cholesterol synthesis, cholesteryl ester transfer protein (CETP) activity and tissue specific editing of apolipoprotein (apo) B mRNA and secretion of apo B-100 from the liver and apo B-48 from the small intestine. Hamsters, like humans, take up approximately $80 \%$ of LDL-C via the LDL receptor pathway. The morphology of aortic foam cells and lesions in hamsters fed atherogenic diets was reported to be similar to human lesions [70]. Recently, in a systematic review Dillard and colleagues concluded that the Golden-Syrian hamster does not appear to be a constructive model to determine the mechanism(s) of diet-induced development of atherosclerotic lesions (see review [71]) however the authors only concentrated on atherogenecity of cholesterol- and fat-rich diets in hamster models of atherosclerosis.

Leung and colleagues investigated intestinal lipoprotein production and the response to insulin sensitization in the high fat-fed Syrian Golden hamster for 5 weeks [72]. They concluded that Syrian Golden Hamsters were fed $60 \%$ fat is a good model of nutritionallyinduced IR that intestinal overproduction of lipoproteins appear to contribute to the hypertriglyceridemia of IR in this animal model and insulin sensitization with rosiglitazone (an insulin sensitizer) ameliorates intestinal apoB48 particle overproduction in this model. An appropriate dyslipidemic animal model that has diabetes would provide an important tool for research on the treatment of diabetic dyslipidemia. Ten days of high fat feeding in golden Syrian hamsters resulted in a significant increase in IR and baseline serum lipid levels accompanied by a prominent dyslipidemia. Thirteen days of treatment with fenofibrate, a peroxisome proliferator-activated receptor alpha (PPAR alpha) selective agonist, produced a dose-dependent improvement in serum lipid levels characterized by lowered VLDL-C and LDL-C and raised HDL-C in a fashion similar to that seen in man [73]. Various diet formula, fat resources and time tables have been found to induce some aspects of MetS in the literature. For example, a diet consisted of $80 \mathrm{~g}$ of anhydrous butterfat, $100 \mathrm{~g}$ of corn oil, $20 \mathrm{~g}$ of Menhaden fish oil and $1.5 \mathrm{~g}$ of cholesterol has been used to encourage hypercholesterolemia in male golden Syrian hamsters for 21 days [68]. Male golden hamsters were given $15 \%$ HFD contained $100 \mathrm{~g}$ of lard and $50 \mathrm{~g}$ of soybean oil and $100 \mathrm{~g}$ of sucrose showed diabetic dyslipidemia for eight weeks [74]. F1B hamster is a geneticallydefined hamster, derived from two highly inbred lines, namely by crossbreeding between Bio 87.20 female with a Bio 1.5 male. F1B hamster is an exciting animal model for hyperlipidemic-related applications. The F1B strain is very responsive to SF and cholesterol by increasing the non-HDL fraction to a greater extent than the HDL fraction [75]. Dietary 
fatty acid chain length, degree of saturation and cis-trans conformation have been shown to alter several metabolic pathways involving cholesterol throughout the body, the combined effect of which is reflected in plasma lipid and lipoprotein profiles (see review [76]). Interestingly, intake of trans-fatty acids in shortenings and margarines has been linked to increased risk of cardiovascular disease through effects on lipoprotein metabolism and substituting trans-fatty acids for either saturated or polyunsaturated fatty acids results in more deleterious lipid-lipoprotein profiles [77]. Hamsters are candidate model to investigate cardiometabolic risks of different fat resource and fat-rich diets [78]. Similarity with the human LDL receptor gene, makes hamster ideal to study LDL receptor antagonists and also useful for drugs which interfere with CETP activities and reverse cholesterol transport (RCT) from peripheral tissues to the liver for biliary and fecal excretion [79]. A considerable amount of experimental attention is currently directed at understanding the in vivo mechanisms of RCT. Although not established in vivo, RCT is thought to be impaired in patients with MetS, in which liver steatosis prevalence is relatively high. In this sense, Briand and coworkers [80] introduced a hamster model of MetS to study RCT. These scientists with the help of HFT diet containing $27 \%$ fat, $0.5 \%$ cholesterol, and $0.25 \%$ deoxycholate as well as $10 \%$ fructose in drinking water for 4 weeks induced promoted IR, dyslipidemia with significantly higher plasma non-HDL-C concentrations and CETP activity, and hepatic steatosis. In vivo RCT was assessed by intraperitoneally injecting (3)Hcholesterol labeled macrophages. Finally their results indicate a significant increase in macrophage-derived cholesterol fecal excretion, which may not compensate for the dietinduced dyslipidemia and liver steatosis [80]. One of the main target organs of MetS is liver, in which it manifests itself as NAFLD [81]. Bhathena and colleagues currently developed a triumphant BioF1B Golden Syrian hamster model of MetS that successfully manifested hyperlipidemia, IR and NAFLD [81]. They induced this model by feeding hamsters a highfat, high-cholesterol, inadequate methionine- and choline-containing diet. In addition to F1B hamster strain from Biobreeders (Watertown, MA) that commonly used to study dietinduced metabolic disorders other three outbred strains are Charles River (CR), Sasco and Harlan (see review [71]). All these strains are derived from inbred or outbred Golden-Syrian hamster.

Similar to rats, hamsters fed high fructose diets ( $60 \%$ of energy) may develop IR and hypertriglyceridemia in TG after only two weeks compared to diets low in fructose [72,73]. Interestingly, hamsters fed high-sucrose diets did not have elevated TG levels and developed only mild IR relative to those fed diets high in fructose [72]. Avramoglu and colleagues reviewed mechanisms of metabolic dyslipidemia in insulin resistant states (see review [82]). They developed an explanatory fructose-fed hamster model of insulin resistance to study hepatic lipid metabolism as its lipoprotein metabolism as described previously $[83,84]$. Hamsters exhibit obesity, hypertriglyceridemia, increase plasma FFAs concentration and IR if fed fructose-rich diet for a two week period. Fructose feeding induced a noteworthy increase in synthesis and secretion of total TGs as well as VLDL-TG by primary hamster hepatocytes [73]. The microsomal triglyceride transfer protein plays a pivotal role in VLDL assembly and its activity showed a striking 2.1-fold elevation in 
hepatocytes derived from fructose-fed versus control hamsters [73]. The apoB production also has been increased in the fructose-fed hamsters [73]. Fructose-fed hamster also has been introduced as an exploratory animal model to excavate role of intestinal lipoprotein overproduction in the dyslipidemia of insulin-resistant states [74]. The authors have shown that fructose feeding for 3 weeks increases secretion of apoB48-containing lipoproteins in the fasting state and during steady state fat feeding. Wang and coworkers [75] investigated the composition of plasma lipoproteins in hamsters fed high-carbohydrate diets of varying complexity ( $60 \%$ carbohydrate as chow, cornstarch, or fructose) for 2 weeks. They showed that hamsters fed the high-fructose diet showed significantly increased VLDL-triglyceride $(92.3 \%)$, free cholesterol (68.6\%), and phospholipid (95\%), whereas apolipoprotein B levels remained unchanged. Fructose feeding induced a $42.5 \%$ increase LDL-triglyceride concurrent with a $20 \%$ reduction in LDL-cholesteryl ester. Compositional changes were associated with reduced LDL diameter. In contrast, fructose feeding caused elevations in all HDL fractions.

\subsection{The guinea pig models}

A number of seminal reviews on the details of the criteria that make guinea pigs suitable animal models for studying lipoprotein metabolism are available (see reviews [85-87]) and a summary will be presented here. Guinea pigs in contrast to other rodents have higher levels of plasma LDL-C compared to HDL-C. As humans, guinea pigs have higher concentrations of free compared to esterified cholesterol found in the liver and they show evidence of moderate rates of hepatic cholesterol synthesis and catabolism. Similar to humans, the binding domain for the LDL receptor of guinea pigs discriminates normal and familial binding defective apo B-100 and apo B mRNA editing in liver is scarce $(<1 \%)$ compared to 18 to $70 \%$ in other species [88]. The three important proteins involved in lipoprotein remodeling and RCT (CETP, lecithin:cholesterol acyltransferase (LCAT), and lipoprotein lipase (LPL) have been reported in guinea pigs.

Guinea pigs have been used as models to dissect the mechanisms by which various dietary fat resources influences plasma lipid-lipoprotein profiles. In contrast to hamsters they do not possess a fore-stomach fermentation which modifies dietary macronutrients before reaching the small intestine [89]. Guinea pigs are not only superior models for studying the mechanisms by which statins [90], cholestyramine [91], apical sodium bile acid transport inhibitors [92] and microsomal transfer protein inhibitors [93] lower plasma LDL-C but also are selected to investigate the mechanisms by which certain drugs or toxins affect lipidlipoprotein metabolism (e.g.,[94]). Guinea pigs respond to dietary fat saturation, dietary cholesterol and dietary fiber by alterations in LDL-C (see review [87]). For example, the SFrich diet will increase TC and LDL-C levels much more than polyunsaturated fat (PF)-rich diet in guinea pigs and cholesterol-rich diets can further increase TC and LDL-C levels [95].

The suitability of guinea pigs as models of atherosclerosis is augmented by an array of review and assessment features (see review [59]). However, guinea pigs do not develop advanced atherosclerotic lesions, and are not an entrenched model for atherosclerosis progression [96]. 
High plasma level of lipoprotein (a) (also called Lp(a)) is associated with coronary heart disease and other forms of atherosclerosis in humans (see review [97]), and as primates, hedgehogs [98] and guinea pigs possess Lp(a) among normal animal models [99]. Guinea pigs are practical model to study the role of oxidized LDL (oxLDL) in progression of atherosclerosis [100]. Initial atherosclerosis induced by various formula of HFD in guinea-pigs. Intake of HFD (guinea-pig pellet diet $+0.2 \% \mathrm{w} / \mathrm{w}$ cholesterol) can induce the onset of early atherosclerotic changes in coronary artery, aorta and major organs at least for one month [101]. High SF diet supplemented with high cholesterol $(0.25 \%)$ will advance an atherosclerotic process for twelve weeks in guinea pigs [102]. Yang and colleagues [103] introduced a hyperlipidemic guinea pig model in a comparative investigation. They concluded that different response of TG metabolism to a HFD $(0.1 \%$ cholesterol and $10 \%$ lard $)$ in guinea pigs and rats suggests that Hartley guinea pigs could be a better hypertriglyceridemia animal model than rats for research on lipid metabolism disorders and hypolipidemic drugs. It seems that chronic dyslipidemia associated with hypertriglyceridemia may reduce auditory function. In this context guinea pigs fed a HFD used as an animal model to find the relationship between of sensorineural hearing loss and dyslipidemia [104].

As rats, guinea pigs are accepted models for studying fetal programming of cardiovascular diseases. Interestingly, adipogenesis in the guinea pig is very active during early postnatal life and was altered by a maternal HFD; thus, it is an adequate model for intrauterine fat deposition [105]. More studies are requested to explore lipid-lipoprotein profiles of guinea pigs that received a maternal HFD. Caillier and colleagues [106] currently generated a guinea pig model of MetS by 150-day exposure to diabetogenic high fat high sucrose or the high fat high fructose diets. To my knowledge, it would be early to consider guinea pig as an animal model of MetS since the literatures are scarce.

\subsection{The rabbit models}

A century ago, rabbits were used as translated animal models of atherosclerosis [107]. Since then, a number of animal models have been used to explain the relationship between disorders of lipid metabolism and atherogenesis (see reviews [108,109]). In this sense, dietary lipid manipulation and use of naturally defective animals, such as Watanabe heritable hyperlipidemia (WHHL) rabbits, have been the focus of most experimental settings (see chapter 22). Rabbits are appropriate animal models for studying lipoprotein metabolism and its disorders because they share with humans several aspects of lipoprotein metabolism, such as similarities in composition of apolipoprotein B containing lipoproteins, hepatic production of apo B 100-containing VLDL, plasma CETP activity, human-like apo B, low hepatic lipase activity and high absorption rate of dietary cholesterol. Unlike humans, rabbits are hepatic lipase-deficient and do not have an analogue of human apo A-II. Rabbits do not form spontaneous atherosclerotic lesions and therefore require very high cholesterol levels to induce more advanced disease (see review [110]). Rabbits also have significant differences in their lipid metabolism from humans, which can result in their development of "cholesterol storage syndrome" while on high-cholesterol diets (0.5-3\%), with cholesterol deposited in their liver, adrenal cortex, and reticuloendothelial and genitourinary systems 
[108]. We found that a high-cholesterol diet contained in $0.47 \%$ cholesterol would be tolerable for adult male rabbits for 4 weeks but our high-cholesterol diet was mildly atherogenic [94,111]. The atherosclerotic lesions of rabbits do not completely resemble those in humans [108] and the formed lesions are more fatty and macrophage rich than human [112]. Atherogenic diets are usually associated with hypercholesterolemia and the development of atherosclerotic lesions in the aortic arch and thoracic aorta rather than in the abdominal aorta that is almost always affected in humans. New Zealand White rabbits are the strain commonly used in atherosclerosis research. Although they have low plasma TC concentrations and HDL as dominant lipoprotein [113], ${ }_{\beta}$ VLDL becomes the major class of plasma lipoproteins when exposed to cholesterol-rich diet (see review [59]). In conjunction with chylomicron remnants, $\beta$ VLDL becomes highly atherogenic. Long-term experiments using diets high in cholesterol are discouraging in rabbits, because they cannot increase the excretion of sterols and resulting hepatotoxicity does not allow the animal to survive (see review [114]).

Various HFD and intervention period have been used to induce MetS in rabbits or its components like IR, visceral obesity, hypertension, dyslipidemia (e.g., [115,116]). Rabbits are suitable animals to investigate MetS-associated multiorgan dysfunctions. Helfenstein and colleagues recently proposed an experimental model of impaired glucose tolerance combined with hypercholesterolaemia induced by diets (high-fat/high-sucrose (10/40\%) and cholesterolenriched diet for 24 weeks) that gained weight, increased blood glucose, TC, LDL-C, TGs, and decreased HDL-C in New Zealand male rabbits [117]. Their cheap model reproduced several metabolic characteristics of human DM and promoted early signs of retinopathy. Corona and colleagues [118] reviewed relationships between hypogonadism and MetS emphasizing their possible interaction in the pathogenesis of cardiovascular diseases. However they concluded that the clinical significance of the MetS-associated hypogonadism needs further clarifications. Vignozzi and colleagues [119] described an animal model of MetS obtained by feeding male rabbits for 12 weeks. In their experiment, HFD-animals develop hypogonadism and all the MetS features like hyperglycemia, glucose intolerance, dyslipidemia, hypertension, and visceral obesity. A recently established rabbit model of HFD-induced MetS showed hypogonadism and the presence of prostate gland alterations, including inflammation, hypoxia and fibrosis [120]. Rabbits fed a cholesterol-rich diet (1\% cholesterol) for 8 weeks and 12 weeks share several physiopathological aspects of NAFLD [121]. Because this model is not insulin resistant and obese, it may be useful for elucidating the mechanism of NAFLD related mainly to hyperlipidemia.

\section{Spontaneous animal models of lipoprotein disorders}

The pathophysiology of disorders of lipoprotein metabolism of humans cannot highly translated to wild-type rodents since they are very resistant to atherogenesis and have no similarity to human lipid and lipoprotein metabolism; further, they do not develop cardiovascular diseases identical to humans. Therefore search for more reliable model is still continuing. In this regard, pig, is a considered a very good model of human atherosclerosis, because it is similar to humans in terms of body size and other physiological features (see 
review [122]). Pigs spontaneously develop atherosclerosis even on a normal porcine diet and dietary modification lead to sever atherosclerosis [123]. As humans, pigs transport most cholesterol in LDL-C and dietary modification alters their plasma lipoproteins closely resemble those occurring in humans. In contrast to rodents, swine atherosclerosis, like the human illness, progresses to advanced stages (see review [122]). For example, mini-pigs fed fat-enriched food showed fatty streaks in their abdominal and thoracic aorta and coronary arteries during 18 months [124]. Cholesterol contents of diets also affects the extension and exacerbation of atherosclerotic changes in pigs (see review [122]). Johansen and colleagues [125] suggested an obese Göttingen minipig model of MetS that was highly responsive to a high fat high energy diet. Several swine models of T2D and IR have been proposed (see review [18]). However, spontaneity in development of MetS and IR, is not common in this species [58]. Dogs do not naturally show atherosclerosis and cholesterol- and SF-rich diets combined with thyroid suppression is required for atherosclerosis development [126]. Beagles and miniature Schnauzer dogs show useful similarities with human in cholesterol synthesis, and lipoproteins level [127,128]. Feline DM, in both spontaneous and inducible forms, therefore provides a reliable animal model of human T2D and may provide additional insights into the clinical, physiological, and pathological features of this disease (see review [129]). Considerably more studies must be forthcoming to establish firmly how lipoprotein profile participates in pathogenesis of atherosclerosis, DM and possibly MetS in pet animal since companion animal obesity would be a serious veterinary medical concern in near future [130]. The subject of suitability of other domesticated animal species such as pigs and sheep, as well as feral, migrating and hibernating species for studying lipid and lipoprotein metabolism has been concisely reviewed (see review [131]). For thorough coverage of this aspect of animal models, this work is recommended.

A number of other interesting wild rodents that are explanatory or exploratory animal models of different disorders of lipid and/or carbohydrate metabolism have been introduced. Recently, Octodon degus (degu) has been proposed as an animal model of dietinduced development of atherosclerosis [132]. To induce atherosclerosis, degus were fed for 16 weeks chow containing $0.25 \%$ cholesterol and $6 \%$ palm oil. Cholesterol-fed degus exhibited 4- to 5-fold increases in TC, principally in the VLDL-C and LDL-C fractions and developed cholesteryl ester-rich atherosclerotic lesions throughout the aorta [132]. Hedgehogs are homologous animal models for studying roles of $\mathrm{Lp}(\mathrm{a})$ in atherosclerosis [98]. Sand rat, Tuco-Tuco and spiny mouse are unusual models of diet-induced obesity and T2D (see review [12]. In laboratory condition, sand rat (Psammomys obesus) develops obesity and diabetes when fed on standard chow (high energy diet) instead of its usual energydiluted vegetable diet composed mainly of saltbush Atriplex [133]. Surprisingly, sand rats are studied extensively and serve as more statable polygenic model for the study of diabesity syndrome $[133,134]$. Spiny mouse (Acomys calirinus) is another small rodent living in semiarid areas of eastern Mediterranean. They gain weight and exhibit marked impaired pancreatic beta cell when they are placed in captivity on high energy rodent lab chow [135]. Ctenomis talarum (Tuco-tuco) is another feral species which exhibit similar characteristic features of sand rat and spiny mice when fed on high energy rodent diet [136]. Brandt's vole 
(Lasiopodomys brandtii) is another rodent model used in diet-induced obesity [137]. The Nile grass rat (NGR), Arvicanthis niloticus, is a herbivorous rodent inhabiting dry savanna, woodlands, and grasslands in Africa. Noda and colleagues [138] recently showed that the NGR is a precious, spontaneous model for exploring the etiology and pathophysiology of MetS as well as its various complications.

Avian models of human atherosclerosis include pigeon, chicken, Japanese quail, turkey and parrots. Although these avian models are not frequently used in studying atherosclerosis, it is worthy to note, that spontaneous atherosclerosis in the chicken was first described in 1914 [139]. Use of pigeon models of atherosclerosis has been extensively reviewed (see review [140]). Briefly, the most important key points supporting the use of pigeons as models for human atherosclerosis include: 1. Pigeons are hypercholesterolemic compared to humans. 2. Pigeons are primarily HDL-C carriers but $\beta$ VLDL-C and LDL-C become major lipid carriers when these animals are fed cholesterol-rich diet. 3. Their lipid metabolism and lesion progression are similar to humans. 4. Pigeons also resemble humans in cellular and vascular dysfunctions involving in atherogenesis. 5. Pigeons are negative animal models to study relevance of apoE, apo B48, chylomicra or LDL receptor in atherosclerosis pathology. 6 . Pigeons are susceptible to both spontaneous and diet-induced atherosclerosis. Parrots are exceptional animal models to assess the impacts of various risk factors include elevated cholesterol level, diet composition, social stress and inactivity (similar to sedentary behavior in humans) on occurrence and progression of atherosclerosis (see review [59]).

Phylogenetically, nonhuman primates are more similar to humans than other models in terms of lipid-lipoprotein profiles, pathophysiology of atherosclerosis, feeding habits, and genotype. It is demonstrated that, along with aging, some rhesus monkeys spontaneously develop diabesity (e.g. [141]). In this context, Macaca nigra is very valuable in studies focused on the interactions between atherosclerosis and diabetes [142]. Spontaneous diabetes has been documented in nonhuman primates include cynomolgus, rhesus, bonnet, Formosan rock, pig-tailed, celebes macaques, African green monkeys, and baboons (see reviews $[18,143])$. Diabetic nonhuman primates have detrimental changes in plasma lipid and lipoprotein concentrations and lipoprotein composition which may contribute to progression of atherosclerosis. As both the prediabetic condition (similar to MetS in humans) and overt diabetes become better translated in monkeys, their use in pharmacological studies is increasing. Monkeys can be categorized into hyperresponders and hyporesponders based on initiation, progression and severity of atherosclerotic lesions. Several nonhuman primates, such as squirrel monkeys, baboons, and wooly and spider monkeys, may develop spontaneous early stage (fatty streaks) atherosclerosis at different anatomical locations (see reviews $[59,108]$ ). Rare cases of LDL receptor deficiency in a rhesus monkey family associated with increased levels of LDL-C, Lp(a), and advanced atherosclerotic lesions in the aorta, and to a lesser extent in coronary arteries, were reported [144]. Nonhuman primates are more reliable model to study cardiovascular disease plus MetS rather than rodents, since they develop MetS and cardiovascular diseases as they age. They develop spontaneous (in some species) and high fat high cholesterol diet-induced atherosclerotic lesions [145]. Nonhuman primates are good model of hypertension and its 
harmful effect on atherosclerosis development. The close similarity of plasma lipoproteinlipid level, plaque development and its calcification and mineralization with humans makes nonhuman primates practical model to explore the correlation between plasma lipids and plaque development (see review [59]). Kaufman and colleagues measured some anthropometric indices and metabolic parameters in 250 laboratory-born bonnet macaques living in social groups and maintained on commercial monkey chow [146]. Finally they concluded socially reared and housed bonnet macaques may provide a useful model for studying the pathogenesis, prevention, and treatment of the MetS. Recently, in an excellent investigation, Zhang and colleagues established a rhesus monkey model of spontaneous MetS using population screening approaches suitable to explore the pathogenesis of MetS in relation to cardiovascular disease and DM [147]. To sum, the inconsistency in anatomic location of atherosclerotic lesions, high cost of husbandry and veterinary services, limited animal availability, difficult handling, together with ethical queries are major obstacles in the use of monkeys as common animal models in studying MetS and its comorbidities.

\section{Conclusion}

The incidence of metabolic syndrome is increasing on a pandemic level. One of the major underlying cause and/or outcome of metabolic syndrome is dyslipidemia, which contribute greatly to the cardiovascular problems associated with the syndrome. The animal models have a vital role to play in extending our understanding of metabolic syndrome and its related comorbidities. Conventional laboratory animals such as mice, rats, hamsters, guinea pigs and rabbits have been examined to gain a better perceptive of the relationship between disorders of lipid metabolism and their clinical correlations. High-fat diets frequently used to induce different aspects of metabolic syndrome in rodent models. However, nonconventional animal models like pig, pigeon, and feral animals (e.g., spiny mice, sand rat, hedgehogs) can consider as spontaneous animal models suitable for studying both the pathogenesis and potential therapeutic agents in lipoprotein disorders. The attempts to find animal models relevant to the study of metabolic syndrome are continuing.

\section{Author details}

Isaac Karimi

Division of Biochemistry, Physiology and Pharmacology, Department of Basic Veterinary Sciences, School of Veterinary Medicine, Razi University, Kermanshah, Iran

\section{References}

[1] Joslin EP (1922) The prevention of diabetes mellitus. JAMA. 76(2):79-84.

[2] Vague J (1956) The degree of masculine differentiation of obesities: a factor determining predisposition to diabetes, atherosclerosis, gout, and uric calculous disease. Am J Clin Nutr 4: 20-34. 
[3] Haller H (1977) Epidermiology and associated risk factors of hyperlipoproteinemia. Z Gesamte Inn Med. 15;32(8):124-8. [Article in German]

[4] Phillips GB (1978) Sex hormones, risk factors and cardiovascular disease. Am J Med. 65:7-11.

[5] Phillips GB (1977) Relationship between serum sex hormones and glucose, insulin, and lipid abnormalities in men with myocardial infarction. Proc Natl Acad Sci USA.74: 1729-1733.

[6] Reaven GM (1988) Banting lecture 1988. Role of insulin resistance in human disease. Diabetes 37: 1595-1607.

[7] World Health Organization (1999) WHO consultation: definition, diagnosis and clasification of diabetes mellitus and its complications. Geneva: WHO.

[8] Alberti KG, Zimmet P, Shaw J (2005) The metabolic syndrome: a new worldwide definition. Lancet. 24; 366:1059-62.

[9] Balkau B, Charles MA (1999) Comment on the provisional report from the WHO consultation. European Group for the Study of Insulin Resistance (EGIR). Diabet Med.16:442-3.

[10] Executive Summary of The Third Report of The National Cholesterol Education Program (NCEP) (2001) Expert Panel on Detection, Evaluation, And Treatment of High Blood Cholesterol In Adults (Adult Treatment Panel III). JAMA.285:2486-97.

[11] Cziraky MJ (2004) Management of dyslipidemia in patients with metabolic syndrome. J Am Pharm Assoc. 44: 478-488.

[12] Srinivasan K, Ramarao P (2007) Animal models in type 2 diabetes research: An overview. Indian J Med Res. 125: 451-472

[13] Weiss R, Dziura J, Burgert TS, Tamborlane WV, Taksali SE, Yeckel CW, Allen K, Lopes M, Savoye M, Morrison J, Sherwin RS, Caprio S (2004) Obesity and the metabolic syndrome in children and adolescents. N Engl J Med.350:2362-74.

[14] Bruce KD, Byrne CD (2009) The metabolic syndrome: common origins of a multifactorial disorder. Postgrad Med J. 85:614-621.

[15] Getz GS, Reardon CA (2006) Diet and murine atherosclerosis. Arterioscler Thromb Vasc Biol. 26:242-249.

[16] Maxwell KN, Soccio RE, Duncan EM, Sehayek E, Breslow JL (2003) Novel putative SREBP and LXR target genes identified by microarray analysis in liver of cholesterolfed mice. J Lipid Res. 44:2109-2119.

[17] Nishina PM, Lowe S, Verstuyft J, Naggert JK, Kuypers FA, Paigen B (1993) Effects of dietary fats from animal and plant sources on diet-induced fatty streak lesions in C57BL/6J mice. J Lipid Res. 34:1413-1422.

[18] Cefalu WT (2006) Animal models of type 2 diabetes: clinical presentation and pathophysiological relevance to the human condition. ILAR J. 47(3).

[19] Panchal SK, Brown L (2011) Rodent models for metabolic syndrome research. J Biomed Biotechnol. 2011:351982.

[20] Kobayasi R, Akamine EH, Davel AP, Rodrigues MAM, Carvalho CRO and Rossoni LV (2010) "Oxidative stress and inflammatory mediators contribute to endothelial dysfunction in high-fat diet-induced obesity in mice," J Hypertension. 28: 2111-2119. 
[21] Deji N, Kume S, Araki SI et al (2009) "Structural and functional changes in the kidneys of high-fat diet-induced obese mice,". Am J Physio. 296: F118-F126.

[22] Buettner R, Parhofer KG, Woenckhaus M, Wrede CE, Kunz-Schughart LA, Scholmerich J, Bollheimer LC (2006) Defining high-fat-diet rat models: metabolic and molecular effects of different fat types. J Mol. Endocrinol. 36:485-501.

[23] Sutherland LN, Capozzi LC, Turchinsky NJ, Bell RC, Wright DC (2008) “Time course of high-fat diet-induced reductions in adipose tissue mitochondrial proteins: potential mechanisms and the relationship to glucose intolerance," Am J Physio. 295: E1076E1083.

[24] Sweazea KL, Lekic M, Walker BR (2010) "Comparison of mechanisms involved in impaired vascular reactivity between high sucrose and high fat diets in rats," Nutr Metabol. 7:48.

[25] Basciano H, Federico L, Adeli K (2005) Fructose, insulin resistance, and metabolic dyslipidemia. Nutr Metabol. 2:5 doi:10.1186/1743-7075-2-5.

[26] Kohli R, Kirby M, Xanthakos SA, Softic S, Feldstein AE, Saxena V, Tang PH, Miles L, Miles MV, Balistreri WF, Woods SC, Seeley RJ (2010) High-fructose, medium chain trans fat diet induces liver fibrosis and elevates plasma coenzyme Q9 in a novel murine model of obesity and nonalcoholic steatohepatitis. Hepatology. 52(3):934-44.

[27] Nascimento FA, Barbosa-da-Silva S, Fernandes-Santos C, Mandarim-de-Lacerda CA, Aguila MB (2010) Adipose tissue, liver and pancreas structural alterations in C57BL/6 mice fed high-fat-high-sucrose diet supplemented with fish oil (n-3 fatty acid rich oil). Exp Toxicol Pathol. 62(1):17-25.

[28] Charlton M, Krishnan A, Viker K, Sanderson S, Cazanave S, McConico A, Masuoko H, Gores G (2011) Fast food diet mouse: novel small animal model of NASH with ballooning, progressive fibrosis, and high physiological fidelity to the human condition. Am J Physiol Gastrointest Liver Physiol. 301(5):G825-34.

[29] Surwit RS, Kuhn CM, Cochrane C, McCubbin JA, Feinglos MN (1988) Diet-induced type II diabetes in C57BL/6J mice. Diabetes. 37: 1163-7.

[30] Ikegami H, Fujisawa T, Ogihara T (2004) Mouse models of type 1 and type 2 diabetes derived from the same closed colony: Genetic susceptibility shared between two types of diabetes. ILAR J. 45:268-277.

[31] Harmon JS, Gleason CE, Tanaka Y, Poitout V, Robertson RP (2001) Antecedent hyperglycemia, not hyperlipidemia, is associated with increased islet triacylglycerol content and decreased insulin gene mRNA level in Zucker diabetic fatty rats. Diabetes. 50:2481-2486.

[32] Hu Y, Davies GE (2010) Berberine inhibits adipogenesis in high-fat diet-induced obesity mice. Fitoterapia. 81: 358-366.

[33] Levin BE, Triscari J, Sullivan AC (1983) Relationship between sympathetic activity and diet-induced obesity in two rat strains. Am J Physiol. 245: R364-R371.

[34] Lauterio TJ, Bond JP, Ulman EA (1994) Development and characterization of a purified diet to identify obesity-susceptible and -resistant rat populations. J Nutr. 124:2172-2178.

[35] Chang S, Graham B, Yakubu F, Lin D, Peters JC, Hill JO (1990) Metabolic differences between obesity-prone and obesity-resistant rats. Am J Physiol 259:R1103-R1110. 
[36] Levin BE, Dunn-Meynell AA (2006) Differential effects of exercise on body weight gain and adiposity in obesity-prone and -resistant rats. Int J Obes(Lond). 30:722-727.

[37] Levin BE, Keesey RE (1998) Defense of differing body weight set points in diet-induced obese and resistant rats. Am J Physiol. 274:R412-R419.

[38] Dobrian AD, Davies MJ, Prewitt RL, Lauterio TJ (2000) Development of Hypertension in a Rat Model of Diet-Induced Obesity. Hypertension.35:1009-1015.

[39] Krauss RM (2004) Lipids and lipoproteins in patients with type 2 diabetes. Diabetes Care.27:04-1496.

[40] Monti LD, Landoni C, Setola E, Galluccio E, Lucotti P, Sandoli EP, Origgi A, Lucignani G,Piatti P, Fazio F (2004) Myocardial insulin resistance associated with chronic hypertriglyceridemia and increased FFA levels in Type 2 diabetic patients. Am J Physiol Heart Circ Physiol. 287:H1225-31.

[41] Akiyama T, Tachibana I, Shirohara H, Watanabe N, Otsuki M (1996) High-fat hypercaloric diet induces obesity glucose intolerance and hyperlipidemia in normal adult male Wistar rat. Diabetes Res Clin Pract. 31: 27-35.

[42] Lauterio TJ, Barkan A, DeAngelo M, DeMott-Friberg R, Ramirez R (1998) Plasma growth hormone secretion is impaired in obesity-prone rats before onset of dietinduced obesity. Am J Physiol. 275:E6-E11.

[43] Yang N, Wang C, Xu M, Mao L, Liu L, Sun X (2005) Interaction of dietary composition and PYY gene expression in diet-induced obesity in rats. J Huazhong Univ Sci Technolog Med Sci.25:243-6.

[44] Barker DJP, Gluckman PD, Godfrey KM, Harding JE, Owens JA, Robinson JS (1993) Fetal nutrition and cardiovascular disease in adult life. Lancet 341: 938-941.

[45] Bertram CE, Hanson MA (2001) Animal models and programming of the metabolic syndrome. Brit Med Bull. 60:103-121

[46] Guo F, Jen KLC (1995) High fat feeding during pregnancy and lactation affects offspring metabolism in rats. Physiol Behav.57: 681-6

[47] Brown SA, Rogers LK, Dunn JK, Gotto AM, Jr, Patsch W (1990) Development of cholesterol homeostatic memory in the rat is influenced by maternal diets. Metab Clin Exp. 39: 468-73.

[48] Langley-Evans SC (1996) Intrauterine programming of hypertension in the rat: nutrient interactions. Comp Biochem Physiol. 114: 327-31

[49] Ricci MR, Levin BE (2003) Ontogeny of diet-induced obesity in selectively bred Sprague-Dawley rats. Am J Physiol Regul. Integr. Comp Physiol. 285:R610-R618.

[50] Corsetti JP, Sparks JD, Peterson RG, Smith RL, Sparks CE (2000) Effect of dietary fat on the development of non-insulin dependent diabetes mellitus in obese Zucker diabetic fatty male and female rats. Atherosclerosis. 148:231-241.

[51] Owens D (2006) Spontaneous, surgically and chemically induced models of disease. In The Laboratory Rat. Suckow MA, Weisbroth SH, Franklin CL, Eds. Elsevier Academic Press. 711-732 p.

[52] Blay M, Peinado-Onsurbe J, Julve J, Rodríguez V, Fernández-López JA, Remesar X, Alemany M (2001) Anomalous lipoproteins in obese Zucker rats. Diabetes Obes Metab. 3(4):259-70. 
[53] Ballal K, Wilson CR, Harmancey R, Taegtmeyer H (2010) Obesogenic high fat western diet induces oxidative stress and apoptosis in rat heart. Mol Cell Biochem. 344(1-2):22130.

[54] Hegsted DM, Ausman LM, Johnson JA, Dallal GE (1993) Dietary fat and serum lipids: an evaluation of the experimental data. Am J Clin Nutr. 57:875-883.

[55] Fillios LC, Andrus SB, Mann GV, Stare FJ (1956) Experimental production of gross atherosclerosis in the rat. J Exp Med. 104: 539.

[56] Zaragoza C, Gomez-Guerrero C, Martin-Ventura JL, Blanco-Colio L, Lavin B, Mallavia B, Tarin C, Mas S, Ortiz A, Egido J (2011) Animal models of cardiovascular diseases. J Biomed Biotechnol. doi:10.1155/2011/497841

[57] Russell JC, Proctor SD (2006) Small animal models of cardiovascular disease: tools for the study of the roles of metabolic syndrome, dyslipidemia, and atherosclerosis. Cardiovasc Pathol. 15: 318-30.

[58] Singh V, Tiwari RL, Dikshit M, Barthwal MK (2009) Models to study atherosclerosis: a mechanistic insight. Curr Vasc Pharmacol. 7: 75-109.

[59] Gomibuchi H, Okazaki M, Iwai S, Kumai T, Kobayashi S, Oguchi K (2007) Development of hyperfibrinogenemia in spontaneously hypertensive and hyperlipidemic rats: a potentially useful animal model as a complication of hypertension and hyperlipidemia. Exp Anim.56: 1-10.

[60] Matos SL, Paula Hd, Pedrosa ML, dos Santos RC, de Oliveira EL, Júnior DAC, Silva ME (2005) Dietary models for inducing hypercholesterolemia in rats. Braz Arch Biol Techn. 48:203-209.

[61] Roberts CK, Liang K, Barnard RJ, Kim CH,Vaziri ND (2004) HMG-CoA reductase, cholesterol 7a-hydroxylase, LDL receptor, SR-B1, and ACAT in diet-induced syndrome X. Kidney Int. 66:1503-1511.

[62] Sampey BP, Vanhoose AM, Winfield HM, Freemerman AJ, Muehlbauer MJ, Fueger PT, Newgard CB, Makowski L (2011) Cafeteria diet is a robust model of human metabolic syndrome with liver and adipose inflammation: comparison to high-fat diet. Obesity.19: 1109-1117.

[63] Manting L, Haihong Z, Jing L, Shaodong C, Yihua L (2011) The model of rat lipid metabolism disorder induced by chronic stress accompanying high-fat-diet. Lipids Health Dis. 10:153.

[64] Spady DK, Woollett LA, Dietschy JM (1993) Regulation of plasma LDL- cholesterol levels by dietary cholesterol and fatty acids. Annu Rev Nutr. 13:355-381.

[65] Pien CS, Davis WP, Marone AJ, Foxall TL (2006) Characterization of diet induced aortic atherosclerosis in Syrian F1B Hamsters. J Exp Anim Sci. 42:65-83, 2006

[66] Alexaki A, Wilson TA, Atallah MT, Handelman G, Nicolosi RJ (2004) Hamsters fed diets high in saturated fat have increased cholesterol accumulation and cytokine production in the aortic arch compared with cholesterol-fed hamsters with moderately elevated plasma non-HDL cholesterol concentrations. J Nutr. 134:410-415.

[67] Vinson JA, Mandarano M, Hirst M, Trevithick JR, Bose P (2003) Phenol antioxidant quantity and quality in foods: beers and the effect of two types of beer on an animal model of atherosclerosis. J Agric Food Chem. 51(18):5528-33. 
[68] Rimando AM, Nagmani R, Feller DF, Yokoyama W (2005) Pterostilbene, a new agonist for the peroxisome proliferator-activated receptor r-isoform, lowers plasma lipoproteins and cholesterol in hypercholesterolemic hamsters. J Agric Food Chem. 53: 3403-3407

[69] Nistor A, Bulla A, Filip DA, Radu A (1987) The hyperlipidemic hamster as a model of experimental atherosclerosis. Atherosclerosis. 68:159-173.

[70] Kahlon T, Chow F, Irving D, Sayre R (1996) Cholesterol response and foam cell formation in hamsters fed two levels of saturated fat and various levels of cholesterol. Nutr Res. 16:1353-1368.

[71] Dillard A, Matthan NR, Lichtenstein AH (2010) Use of hamster as a model to study dietinduced Atherosclerosis. Nutr Metab. 7:89

[72] Leung N, Naples M, Uffelman K, Szeto L, Adeli K, Lewis GF (2004) Rosiglitazone improves intestinal lipoprotein overproduction in the fat-fed Syrian Golden hamster, an animal model of nutritionally-induced insulin resistance. Atherosclerosis. 174(2):235-41.

[73] Wang PR, Guo Q, Ippolito M, Wu M, Milot D, Ventre J, Doebber T, Wright SD, Chao YS (2001) High fat fed hamster, a unique animal model for treatment of diabetic dyslipidemia with peroxisome proliferator activated receptor alpha selective agonists. Eur J Pharmacol. 427(3):285-93.

[74] Li S-Y, Chang C-Q, Ma F-Y, Yu C-L (2009) Modulating effects of chlorogenic acid on lipids and glucose metabolism and expression of hepatic peroxisome proliferatoractivated receptor- $\alpha$ in golden hamsters fed on high fat diet. Biomed Environ Sci. 22:122-129.

[75] Tyburczy C, Major C, Lock AL, Destaillats F, Lawrence P, Brenna JT, Salter AM, Bauman DE (2009) Individual trans octadecenoic acids and partially hyrdogenated vegetable oil differentially affect hepatic lipid and lipoprotein metabolism in golden Syrian hamsters. J Nutr.139:257-263.

[76] Kritchevsky D (2001) Diet and atherosclerosis. J Nutr Health Aging. 5:155-159.

[77] Dorfman SE, Laurent D, Gounarides JS, Li X, Mullarkey TL, Rocheford EC, Sari-Sarraf F, Hirsch EA, Hughes TE, Commerford SR (2009) Metabolic implications of dietary trans-fatty acids. Obesity.17:1200-1207.

[78] Costa RRS, Villela NR, Souza MdGS, Boa BCS, Cyrino FZGA, Silva SV, Lisboa PC, Moura EG, Barja-Fidalgo TC, Bouskela E (2011) High fat diet induces central obesity, insulin resistance and microvascular dysfunction in hamsters. Microvascular Res. 82:416-422.

[79] Kothari HV, Poirier KJ, Lee WH, Satoh Y (1997) Inhibition of cholesterol ester transfer protein CGS 25159 and changes in lipoproteins in hamsters. Atherosclerosis. 128: 59-66.

[80] Briand F, Thiéblemont Q, Muzotte E, Sulpice T (2012). High-Fat and Fructose Intake Induces Insulin Resistance, Dyslipidemia, and Liver Steatosis and Alters In Vivo Macrophage-to-Feces Reverse Cholesterol Transport in Hamsters. J Nutr. 142(4):704-9.

[81] Bhathena J, Kulamarva A, Martoni C, Malgorzata A, Urbanska, Malhotra M, Paul A, Prakash S (2011) Diet-induced metabolic hamster model of nonalcoholic fatty liver disease. Diabetes, Metabolic Syndrome and Obesity: Targets and Therapy.4:195-203

[82] Avramoglu RK, Qiu W, Adeli K (2003) Mechanisms of metabolic dyslipidemia in insulin resistant states: deregulation of hepatic and intestinal lipoprotein secretion. Frontiers in Bioscience. 8: 464-476. 
[83] Sullivan MP, Cerda JJ, Robbins FL, Burgin CW, Beatty RJ (1993) The gerbil, hamster, and guinea pig as rodent models for hyperlipidemia. Lab Anim Sci. 43,575-578.

[84] Hoang VQ, Botham KM, Benson GM, Eldredge EE, Jackson B, Pearce N, Suckling KE (1993) Bile acid synthesis in hamster hepatocytes in primary culture: sources of cholesterol and comparison with other species. Biochim Biophys Acta. 1210:73-80.

[85] Fernandez ML(2001) Guinea pigs as models for cholesterol and lipoprotein metabolism. J Nutr.131:10-20.

[86] West KL, Fernandez ML (2004) Guinea pigs as models to study the hypocholesesterolemic effects of drugs. Cardiovasc Rev. 22:7-22.

[87] Fernandez ML, Volek JS (2006) Guinea pigs: a suitable animal model to study lipoprotein metabolism, atherosclerosis and inflammation. Nutr Metab (Lond). 3: 17.

[88] Greeve J, Altkemper I, Dietrich J-H, Greten H, Windler E (1993) Apolipoprotein mRNA editing in 12 different mammalian species: hepatic expression is reflected in low concentrations of apoB-containing plasma lipoproteins. J Lipid Res. 34:1367-1383.

[89] Fernandez ML, Yount NY, McNamara DJ (1990) Whole body cholesterol synthesis in the guinea pig. Effects of dietary fat quality. Biochim Biophys Acta. 1044:340-348.

[90] Madsen CS, Janovitz E, Zhang R, Nguyen-Tran V, Ryan CS, Yin X, Monshizadegan H, Chang M, D'Arienzo C, Scheer S, Setters R, Search D, Chen X, Zhuang S, Kunselman L, Peters A, Harrity T, Apedo A, Huang C, Cuff CA, Kowala MC, Blanar MA, Sun CQ, Robl JA, Stein PD (2008). The Guinea pig as a preclinical model for demonstrating the efficacy and safety of statins. J Pharmacol Exp Ther. 324(2):576-86.

[91] Fernandez ML, Roy S, Vergara-Jimenez M (2000) Resistant starch and cholestyramine have distinct effects on hepatic cholesterol metabolism in guinea pigs fed a hypercholesterolemic diet. Nutr Res.20:837-850.

[92] West K, Ramjiganesh T, Roy S, Keller BT, Fernandez ML (2002) SC-435, an ileal, apical sodium-dependent bile acid transporter inhibitor (ASBT) alters hepatic cholesterol metabolism and lowers plasma low-density-lipoprotein-cholesterol concentrations in guinea pigs. J Pharmacol Exp Theraup.303:291-299.

[93] Aggarwal D, West KL, Zern TL, Shrestha S, Vergara-Jimenez M, Fernandez ML (2005) JTT-130, a microsomal triglyceride transfer protein (MTP) inhibitor lowers plasma triglycerides and LDL cholesterol concentrations without increasing hepatic triglycerides in guinea pigs. BMC Cardiovasc Disord. 27:5:30.

[94] Karimi I, Hayatgheybi H, Shamspur T, Kamalak A, Pooyanmehr M, Marandi Y (2011) Chemical composition and effect of an essential oil of Salix aegyptiaca L. (Musk willow) in hypercholesterolemic rabbit model. Braz J Pharmacog. 21(3): 407-414.

[95] Lin ECK, Fernandez ML, Tosca MA, McNamara DJ (1994) Regulation of hepatic LDL metabolism in the guinea pig by dietary fat and cholesterol. J Lipid Res.35:446-57.

[96] Lynch SM, Gaziano JM, Frei B (1996) Ascorbic acid and atherosclerotic cardiovascular disease. Subcell Biochem. 25: 331-67.

[97] McCormick SPA (2004) Lipoprotein(a): biology and clinical importance. Clin Biochem Rev. 25(1): 69-80.

[98] Hrzenjak A, Frank S, Wo X, Zhou Y, Van Berkel T, Kostner GM (2003) Galactosespecific asialoglycoprotein receptor is involved in lipoprotein (a) catabolism. Biochem J. 376:765-71. 
[99] Rath M, Pauling L (1990) Hypothesis: lipoprotein(a) is a surrogate for ascorbate. Proc Natl Acad Sci USA.87: 6204-7.

[100] Leite JO, DeOgburn R, Ratliff J, Su R, Smyth JA, Volek JS, McGrane MM, Dardik A, Fernandez ML (2010) Low-carbohydrate diets reduce lipid accumulation and arterial inflammation in guinea pigs fed a high-cholesterol diet. Atherosclerosis. 209(2):442-8.

[101] Mangathayaru K, Kuruvilla S, Balakrishna K, Venkhatesh J (2009) Modulatory effect of Inula racemosa Hook. f. (Asteraceae) on experimental atherosclerosis in guinea-pigs. J Pharm Pharmacol. 61(8):1111-8.

[102] Leite JO, Vaishnav U, Puglisi M, Fraser H, Trias J, Fernandez ML (2009) A-002 (Varespladib), a phospholipase A2 inhibitor, reduces atherosclerosis in guinea pigs. BMC Cardiovasc Disord. 17:7.

[103] Yang R, Guo P, Song X, Liu F, Gao N (2011) Hyperlipidemic guinea pig model: mechanisms of triglyceride metabolism disorder and comparison to rat. Biol. Pharm. Bull. 34(7): 1046-1051.

[104] Evans MB, Tonini R, Shope CD, Oghalai JS, Jerger JF, Insull W Jr, Brownell WE (2006) Dyslipidemia and auditory function. Otol Neurotol. 27(5):609-14.

[105] Castañeda-Gutiérrez E, Pouteau E, Pescia G, Moulin J, Aprikian O, Macé K (2011) The guinea pig as a model for metabolic programming of adiposity. Am J Clin Nutr. 94:1838S-1845S

[106] Caillier B, Pilote S, Patoine D, Levac X, Couture C, Daleau P, Simard C, Drolet B (2012) Metabolic syndrome potentiates the cardiac action potential-prolonging action of drugs: a possible 'anti-proarrhythmic' role for amlodipine. Pharmacol Res. 65(3):320-7.

[107] Ignatowski AC (1908) Influence of animal food on the organism of rabbits. Izv Imp Voyenno-Med Akad Peter. 16:154-173.

[108] Moghadasian MH, Frohlich JJ, McManus BM (2001) Advances in experimental dyslipidemia and atherosclerosis. Lab Invest. 81:1173- 1183.

[109] Xiangdong L, Yuanwu L, Hua Z, Liming R, Qiuyan L, Ning L (2011) Animal models for the atherosclerosis research: a review. Protein Cell. 2(3):189-201.

[110] Sider KL, Blaser MC, Simmons CA (2011) Animal models of calcific aortic valve disease. International Journal of Inflammation.ID 364310, 18 pages doi:10.4061/2011/364310.

[111] Karimi I, Hayatgheybi H, Razmjo M, Yousefi M, Dadyan A, Hadipour MM (2010) Anti-hyperlipidaemic effects of an essential oil of Melissa officinalis. L in cholesterol-fed rabbits. J Appl Biologl Sci. 4(1): 23-28.

[112] Badimon L (2001) Atherosclerosis and thrombosis: lessons from animal models. Thromb Haemost. 86: 356-65.

[113] Taylor JM, Fan J (1997) Transgenic rabbit models for the study of atherosclerosis. Front Biosci. 2: d298-308.

[114] Yanni AE (2004) The laboratory rabbit: an animal model of atherosclerosis research. Lab Anim. 38: 246-256.

[115] Morelli A, Vignozzi L, Maggi M, Adorini L (2011) Farnesoid X receptor activation improves erectile dysfunction in models of metabolic syndrome and diabetes. Biochim Biophys Acta. 1812(8):859-66. 
[116] Mallidis C, Czerwiec A, Filippi S, O'Neill J, Maggi M, McClure N (2011) Spermatogenic and sperm quality differences in an experimental model of metabolic syndrome and hypogonadal hypogonadism. Reproduction. 142(1):63-71.

[117] Helfenstein T, Fonseca FA, Ihara SS, Bottós JM, Moreira FT, Pott H Jr, Farah ME, Martins MC, Izar MC (2011) Impaired glucose tolerance plus hyperlipidaemia induced by diet promotes retina microaneurysms in New Zeala6nd rabbits. Int J Exp Pathol. 92(1):40-9.

[118] Corona G, Rastrelli G, Morelli A, Vignozzi L, Mannucci E, Maggi M (2011) Hypogonadism and metabolic syndrome. J Endocrinol Invest. 34(7):557-67.

[119] Vignozzi L, Morelli A, Sarchielli E, Comeglio P, Filippi S, Cellai I, Maneschi E, Serni S, Gacci M, Carini M, Piccinni MP, Saad F, Adorini L, Vannelli GB, Maggi M (2012) Testosterone protects from metabolic syndrome-associated prostate inflammation: an experimental study in rabbit. J Endocrinol. 212(1):71-84.

[120] Morelli A, Comeglio P, Filippi S, Sarchielli E, Cellai I, Vignozzi L, Yehiely-Cohen R, Maneschi E, Gacci M, Carini M, Adorini L, Vannelli GB, Maggi M (2012) Testosterone and farnesoid $\mathrm{X}$ receptor agonist INT-747 counteract high fat diet-induced bladder alterations in a rabbit model of metabolic syndrome. J Steroid Biochem Mol Biol. DOI: 10.1016/j.jsbmb.2012.02.007

[121] Kainuma M, Fujimoto M, Sekiya N, Tsuneyama K, Cheng C, Takano Y, Terasawa K, Shimada Y (2006) Cholesterol-fed rabbit as a unique model of nonalcoholic, nonobese, non-insulin-resistant fatty liver disease with characteristic fibrosis. J Gastroenterol. 41:971-80.

[122] Pasławski R, Pasławska U, Szuba A, Nicpoń J (2011) Swine as a Model of Experimental Atherosclerosis. Adv Clin Exp Med. 20: 211-215.

[123] Royo T, Alfón J, Berrozpe M, Badimon L (2000) Effect of gemfibrozil on peripheral atherosclerosis and platelet activation in a pig model of hyperlipidemia. Eur J Clin Invest. 30(10): 843-852.

[124] Jacobsson L (1998) Experimental hyperlipidemia and atherosclerosis in mini-pigs; influence of certain drugs. Scand J Lab Anim Sci. 25: 85-91.

[125] Johansen T, Hansen HS, Richelsen B, Malmlof R (2001) The obese Göttingen minipig as a model of the metabolic syndrome: Dietary effects on obesity, insulin sensitivity, and growth hormone profile. Comp Med. 51:150-155.

[126] Narayanaswamy M, Wright KC, Kandarpa K (2000) Animal models for atherosclerosis, restenosis, and endovascular graft research. J Vasc Interv Radiol.11: 5-17.

[127] Berkhout TA, Simon HM, Jackson B, Yates J, Pearce N, Groot PH, Bentzen C, Niesor E, Kerns WD, Suckling KE (1997) SR-12813 lowers plasma cholesterol in beagle dogs by decreasing cholesterol biosynthesis. Atherosclerosis. 133: 203-12.

[128] Xenoulis PG, Suchodolski JS, Levinski MD, Steiner JM (2007) Investigation of hypertriglyceridemia in healthy Miniature Schnauzers. J Vet Intern Med. 21: 1224-30.

[129] Henson MS, O'Brien TD (2006) Feline models of type 2 diabetes mellitus. ILAR J. 47: 234-242.

[130] German AJ (2006) The growing problem of obesity in dogs and cats. J Nutr. 136:1940S$1946 S$ 
[131] Clarke I (2008) Models of 'obesity' in large animals and birds. Obesity and Metabolism. 36: 107-117.

[132] Homan R, Hanselman JC, Bak-Mueller S, Washburn M, Lester P, Jensen HE, Pinkosky SL, Castle C, Taylor B (2010) Atherosclerosis in Octodon degus (degu) as a model for human disease. Atherosclerosis. 212(1):48-54.

[133] Shafrir E, Ziv E (1998) Cellular mechanism of nutrionally induced insulin resistance: the desert rodent Psammomys obesus and other animals in which insulin resistance leads to detrimental outcome. J Basic Clin Physiol Pharmacol. 9: 347-85.

[134] Kaiser N, Nesher R, Donath MY, Fraenkel M, Behar V, Magnan C (2005) Psammomys obesus, a model for environment-gene interactions in type 2 diabetes. Diabetes. 54: S137-44.

[135] Velasquez MT, Kimmel PL, Michaelis OE (1990) Animal models of spontaneous diabetic kidney disease. FASEB J. 4: 2850-9.

[136] Weir BJ (1974) The development of diabetes in the tuco-tuco (Ctenomys talarum). Proc R Soc Med. 67(9):843-6.

[137] Zhao Z-J, Chen J-F, Wang D-H (2010) Diet-induced obesity in the short-day-lean Brandt's vole. Physiol Behav. 99(1):47-53.

[138] Noda K, Melhorn MI, Zandi S, Frimmel S, Tayyari F, Hisatomi T, Almulki L, Pronczuk A, Hayes KC, Hafezi-Moghadam A (2010) An animal model of spontaneous metabolic syndrome: Nile grass rat. FASEB J. 24: 2443-2453.

[139] Roberts JC, Jr, Straus R (Eds) (1965) Comparative atherosclerosis; the morphology of spontaneous and induced atherosclerotic lesions in animals and its relation to human disease, Harper \& Row, New York

[140] Anderson JL, Smith SC, Taylor Jr RL (2012) Spontaneous Atherosclerosis in Pigeons: A good model of human disease, atherogenesis, Prof. Sampath Parthasarathy (Ed.), ISBN: 978-953-307-992-9, InTech.

[141] Tigno XT, Gerzanich G, Hansen BC (2004) Age-related changes in metabolic parameters of nonhuman primates. J Gerontol A Biol Sci Med Sci. 59:1081-1088.

[142] Kojic ZZ (2003) Animal models in the study of atherosclerosis. Srp Arh Celok Lek. 131: 266-70.

[143] Wagner JD, Kavanagh K, Ward GM, Auerbach BJ, Harwood Jr HJ, Kaplan JR (2006) Old World Nonhuman Primate Models of Type 2 Diabetes Mellitus. ILAR J. 47: 259-271.

[144] Kusumi Y, Scanu AM, McGill HC, and Wissler RW (1993) Atherosclerosis in a rhesus monkey with genetic hypercholesterolemia and elevated plasma Lp(a). Atherosclerosis. 99:165-174.

[145] Pick R, Johnson PJ, Glick G (1974) Deleterious effects of hypertension on the development of aortic and coronary atherosclerosis in stumptail macaques (Macaca speciosa) on an atherogenic diet. Circ Res. 35: 472-82.

[146] Kaufman D, Smith ELP, Gohil BC, Banerji M-A, Coplan JD, Kral JG, Rosenblum LA (2005) Early Appearance of the metabolic syndrome in socially reared bonnet macaques. J Clin Endocrinol Meta. 90:404-408.

[147] Zhang X, Zhang R, Raab S, Zheng W, Wang J, Liu N, Zhu T, Xue L, Song Z, Mao J, Li K, Zhang H, Zhang Y, Han C, Ding Y, Wang H, Hou N, Liu Y, Shang S, Li C, Sebokova E, Cheng H, Huang PL (2011) Rhesus macaques develop metabolic syndrome with reversible vascular dysfunction responsive to pioglitazone. Circulation. 124(1):77-86. 